\title{
ERRATUM: A TRANSFORMATION OF COOKE'S TREATMENT OF SOME TRIPLE INTEGRAL EQUATIONS $\dagger$
}

\author{
E. R. LOVE and D. L. CLEMENTS $\ddagger$
}

(Received 10 April 1978)

[Editor's Note. The essence of what follows was submitted by E. R. Love at the proof stage, but it was considered impracticable to incorporate it into the paper without delaying that whole issue of the Journal.]

Although the paper has two authors, I wish to exonerate my co-author D. L. Clements from responsibility for the need for these corrections; they affect an aspect of the paper in which he took no part.

The first correction arises from neglecting, in the sixth line on p. 286, to exclude the less usual of the two possibilities occurring in Fredholm's Alternative. The other corrections are needed because the next two paragraphs claim that $f_{+}$and $f_{-}$, and $f_{1}$ and $f_{2}$, have bounded first derivatives; the truth of these matters is expressed in (16.3) and (16.4) below, and entails the subsequent work given.

To fill these gaps, I offer the following in place of the three complete paragraphs on p. 286. It should be remembered that we are discussing a special case of the general equations (1.1)-(1.3) considered in the paper: the case in which $q_{1}(r)=0=q_{2}(r), n=0$ and $\alpha=\frac{1}{2}$.

Since $0<k<1$, the kernels of (15.18) and (15.19) are continuous; and it is easily seen by power series expansions that the right-hand sides of these equations are also continuous in $0 \leqslant x \leqslant k$ if suitably defined at $x=0$. The equations consequently have unique continuous solutions for $f_{+}(x)$ and $f_{-}(x)$ in $0 \leqslant x \leqslant k$ subject to the condition $\|K\|<1$, which is sufficient to exclude the other Fredholm Alternative possibility.

Table A (on p. 274) shows that $\|K\|<1$ whenever $a / b \leqslant 0.86$. But closer inequalities than (10.7) are possible; for instance, $\|K\|<1$ whenever $a / b \leqslant 0.998$ can be proved as follows, when $n=0$ and $\alpha=\frac{1}{2}$. By (10.1), and (9.10) or (9.11),

† This Journal, 19 (1976), 259-288.

‡ Corrections by E. R. Love. 


$$
\begin{aligned}
\|K\|^{2}=\frac{4}{\pi^{2}} \int_{0}^{k} d x \int_{0}^{k} \frac{1}{\left(1-x^{2} t^{2}\right)^{2}} d t & =\frac{4}{\pi^{2}}\left(k^{2}+\frac{2}{3^{2}} k^{6}+\frac{3}{5^{2}} k^{10}+\frac{4}{7^{2}} k^{14}+\ldots\right) \\
& <\frac{4 k^{2}}{\pi^{2}}\left(1+\frac{2}{4.2} k^{4}+\frac{3}{6.4} k^{8}+\frac{4}{8.6} k^{12}+\ldots\right) \\
& =\frac{k^{2}}{\pi^{2}}\left(4+\log \frac{1}{1-k^{4}}\right) .
\end{aligned}
$$

For $a / b=k^{2} \leqslant 0.998$ this shows that $\|K\|^{2}<0.963$. Consequently, the condition of the preceding paragraph is fulfilled for $a / b \leqslant 0.998$, which must surely hold in all practical cases; and so $f_{+}(x)$ and $f_{-}(x)$ then exist and are continuous in $0 \leqslant x \leqslant k$.

Since (15.20) gives, for $0 \leqslant x \leqslant k$, that

$$
f_{1}(c x)=\frac{1}{2}\left\{f_{+}(x)+f_{-}(x)\right\}, \quad f_{2}(c / x)=\frac{1}{2} x\left\{f_{+}(x)-f_{-}(x)\right\},
$$

we can now say that $f_{1}(r)$ is continuous in $0 \leqslant r \leqslant b$ if suitably defined at $r=a$, and $f_{2}(r)$ in $r \geqslant b$ if suitably defined at $r=b$. This establishes what is needed in the first line on p. 285.

From (15.18) and (15.19) we obtain by routine calculations that $f_{+}(x)$ and $f_{-}(x)$ are also differentiable in $0 \leqslant x \leqslant k$ and that

$$
f_{+}^{\prime}(x)=-p_{0} \frac{a+c / k^{2}}{\left(k^{2}-x^{2}\right)^{\frac{1}{2}}}+\psi_{+}(x), \quad f_{-}^{\prime}(x)=-p_{0} \frac{a-c / k^{2}}{\left(k^{2}-x^{2}\right)^{\frac{1}{2}}}+\psi_{-}(x),
$$

where $\psi_{ \pm}(x)$ are continuous in $0 \leqslant x \leqslant k$ (if suitably defined at $x=k$ ). This with (16.2) gives that $f_{1}(r)$ and $f_{2}(r)$ are differentiable in $r<a$ and $r>b$ respectively, with

$$
f_{1}^{\prime}(r)=-\frac{p_{0} r}{\left(a^{2}-r^{2}\right)^{\frac{1}{2}}}+\phi_{1}(r), \quad f_{2}^{\prime}(r)=\frac{p_{0} r}{\left(r^{2}-b^{2}\right)^{\frac{1}{2}}}+\phi_{2}(r),
$$

where $\phi_{1}(r)$ and $\phi_{2}(r)$ are continuous in $r \leqslant a$ and $r \geqslant b$ (if suitably defined at the end-point in each case).

After these preparations we can justify (15.14) and (15.15) (on p. 285). For (15.14) we show that the last term in (15.12) is bounded as $r \rightarrow a-$. Using (16.4) we have, for $r<a$,

$$
\begin{aligned}
\int_{r}^{a} \frac{t\left[f_{1}(t)-f_{1}(a)\right]}{\left(t^{2}-r^{2}\right)^{\frac{1}{2}}} d t & =-\int_{r}^{a} \frac{t}{\left(t^{2}-r^{2}\right)^{\frac{1}{2}}} d t \int_{t}^{a} f_{1}^{\prime}(s) d s \\
& =p_{0} \int_{r}^{a} \frac{t}{\left(t^{2}-r^{2}\right)^{\frac{1}{2}}} d t \int_{t}^{a} \frac{s}{\left(a^{2}-s^{2}\right)^{\frac{1}{2}}} d s-\int_{r}^{a} \phi_{1}(s) d s \int_{r}^{s} \frac{t}{\left(t^{2}-r^{2}\right)^{\frac{1}{2}}} d t \\
& =p_{0} \int_{r}^{a} \frac{\left(a^{2}-t^{2}\right)^{\frac{1}{2}}}{\left(t^{2}-r^{2}\right)^{\frac{1}{2}}} t d t-\int_{r}^{a} \phi_{1}(s) d s \int_{r}^{s} \frac{u}{\left(s^{2}-u^{2}\right)^{\frac{1}{2}}} d u \\
& =p_{0} \frac{\pi}{4}\left(a^{2}-r^{2}\right)-\int_{r}^{a} u d u \int_{u}^{a} \frac{\phi_{1}(s)}{\left(s^{2}-u^{2}\right)^{\frac{1}{2}}} d s
\end{aligned}
$$




$$
\begin{aligned}
& =-\frac{2}{\pi} \frac{1}{r} \frac{d}{d r} \int_{r}^{a} \frac{t\left[f_{1}(t)-f_{1}(a)\right]}{\left(t^{2}-r^{2}\right)^{\frac{1}{2}}} d t \\
& =p_{0}-\frac{2}{\pi} \int_{r}^{a} \frac{\phi_{1}(s)}{\left(s^{2}-r^{2}\right)^{\frac{1}{2}}} d s \\
& =p_{0}+O\left(\left(a^{2}-r^{2}\right)^{\frac{1}{2}}\right) .
\end{aligned}
$$

This is bounded as $r \rightarrow a-$, and so (15.14) is justified. A similar discussion from (15.13) and (16.4) justifies (15.15).

A misprint which escaped correction, although it was also found at the proof stage, occurs in $(14.5)$; there $\left(\rho^{2}-r^{2}\right)^{\beta}$ should be $\left(r^{2}-\rho^{2}\right)^{\beta}$.

\section{Department of Mathematics}

University of Melbourne

Parkville, Vic. 3052 